\section{Is there a reliable association between patient-reported limb claudication and vascular imaging methods in Takayasu arteritis?}

\author{
C.S.R. Araujo, A.M. dos Santos, P.A. Olivo Pallo, R.M.R. Pereira, \\ S.K. Shinjo \\ Division of Rheumatology, Faculdade de Medicina FMUSP, Universidade de Sao Paulo, SP, Brazil
}

\begin{abstract}
SUMMARY
Vessel imaging in Takayasu arteritis (TAK) is often performed in clinical practice following laboratory test abnormalities or clinical symptoms, such as limb claudication. Conversely, the association between limb claudication and vessel imaging manifestations has not been assessed.

This observational, cross-sectional study analyzed 139 adult TAK patients from 2000 to 2018. Their arterial vessel imaging information (especially significant stenosis and occlusion data) was registered and crosschecked with clinical and laboratory data.

When vessel imaging was performed, the median age and disease duration of the patients were 38 (27.3-47.0) and $5.0(1.0-12.0)$ years, respectively. There was no association between arterial abnormalities and demographic data, constitutional symptoms or laboratory parameters. Limb claudication was reported in 42 patients (30.2\%): $17.3 \%$ reported it in the upper left limb (ULL), 12.2\% reported it in the upper right limb (URL), 12.9\% reported it in the lower left limb (LLL), and $12.2 \%$ reported it in the lower right limb (LRL). When crossmatched with imaging, both ULL and URL were associated with left vertebral artery stenosis/occlusion, and URL was associated with right iliac artery stenosis/occlusion, but no other association was found. In contrast, both LLL and LRL claudication were associated with infrarenal aortic, left iliac and right iliac artery stenosis/ occlusion $(\mathrm{p}<0.05)$. Moreover, the ULL and URL claudication symptoms were significantly associated with each other $(\mathrm{p}<0.001)$.

Upper limb claudication was associated only with left vertebral artery stenosis/occlusion, whereas the subclavian arteries were not, suggesting that the symptom might not be fully explained by limb ischemia. In contrast, lower limb claudication was associated especially with infrarenal aortic and/or iliac arteries stenosis/occlusion.
\end{abstract}

Key words: Arterial narrowing; arterial occlusions; systemic vasculitis; vascular limb claudication; vessel imaging.

\section{INTRODUCTION}

akayasu arteritis (TAK) is a rare primary large-vessel vasculitis that affects the aorta and its main branches, as well as the pulmonary artery $(1,2)$. TAK diagnosis involves demographic profiling, vascular signs and symptoms and laboratory and radiological abnormalities (3-9). TA is more common in young women and individuals of Asian ethnicity (2).

The more frequent signs and symptoms of TAK include fever, fatigue, weight loss, absent or diminished pulse, arterial bruit, dis- crepancy in blood pressure between limbs and limb claudication, among others $(3,4)$. Limb claudication is an important symptom that is also included in the 1990 American College of Rheumatology (ACR) TAK criteria classification (6). This symptom is believed to occur due to tissue ischemia on the basis of the rationale concerning patients with intermittent claudication secondary to peripheral arterial disease $(10,11)$. However, TAK usually causes ischemia because of proximal artery narrowing due to vessel inflammation or fibrosis rather than, for instance, the distal atherosclerosis pattern seen
Corresponding author: Samuel Katsuyuki Shinjo Laboratorio de Miopatias Inflamatórias, Division of Rheumatology, Faculdade de Medicina, Universidade de Sao Paulo, CEP 01246-903, Cerqueira Cesar, SP, Brazil E-mail: samuel.shinjo@usp.br 
in peripheral arterial disease $(10,11)$. Therefore, the pathogenesis of limb claudication may be different between these two entities. In clinical practice, TAK disease activity or progression/damage characterization is challenging, because it involves the evaluation and combination of clinical findings as well as laboratory and imaging analysis $(12,13)$. In this context, vessel imaging is performed not only to evaluate possible TAK disease progression or activity, but also to investigate its new signs and symptoms. To date, however, no study has assessed the association between vessel imaging findings and TAK features.

Therefore, this study was aimed to assess the association between possible vessel imaging abnormalities (especially arterial occlusion or stenosis) and TAK features (demographic data, laboratory parameters, and signs and symptoms, especially limb claudication) collected from the medical consultations, that occurred immediately prior to vessel imaging.

\section{PATIENTS AND METHODS}

This was a cross-sectional, single-center study that included 139 consecutive adult patients with TAK from the vasculitis unit of our tertiary-care center. The patients met at least three of the six modified 1990 ACR classification criteria (6). Instead of the arteriogram abnormality criterion, we also considered the abnormal computed tomography angiography or magnetic resonance angiography images of large arterial vessels and their main branches.

The study was approved by the local ethical committee.

From 2000 to 2019, we collected all the images of the aorta and its main branches available in each exam (angiography, computed tomography angiography or magnetic resonance angiography) from TAK patients included in this study. The information on vessel imaging was obtained from electronic reports issued by our hospital's radiology ward. Moreover, we reviewed all images and defined a significant stenosis, whenever the diameter reduction was $>50 \%$ (14). For our analysis, we considered this significant ste- nosis or occlusion of the aorta and its main branches.

We also collected all patients' data, when the vessel exams were requested. These patients' data were obtained from a review of electronic medical records that contained the following previously standardized and parametrized data:

- Demographic data, such as age, gender, and ethnicity;

- TAK disease onset;

- Presence of new signal/symptoms within the past three months symptoms - possibly related to TAK disease - that triggered the imaging evaluation: fatigue or weight loss more than $2 \mathrm{~kg}$, persistent fever more than $38^{\circ} \mathrm{C}$, new and persistent headache, (pre)syncope, bruit over the subclavian artery or aorta, visual abnormalities (e.g., amaurosis), significant or persistent chest or abdominal pain, at least moderate dyspnea, carotidynia, and limb claudication;

- Laboratory data, such as C-reactive protein (CRP, reference value: $<5 \mathrm{mg} / \mathrm{L}$ ), and erythrocyte sedimentation rate (ESR, reference value: $<19 \mathrm{~mm} / 1^{\text {st }}$ hour).

Afterward, the presence or absence of limb vessel claudication was cross-checked with the vessel imaging data.

Statistical analysis. Kolmogorov-Smirnov test was used to evaluate the distribution of each parameter. Data was expressed as mean \pm standard deviation for continuous variables or as frequencies $(\%)$ for categorical variables. The median $\left(25^{\text {th }}-75^{\text {th }}\right.$ interquartile range $)$ was calculated for continuous variables that were non-normally distributed. Comparisons between different parameters were made using the Student's $t$-test or Mann-Whitney test for continuous variables, whereas the chi-squared test or Fisher's exact test was used to evaluate the association between the categorical variables. All of the analyses were performed using the SPSS 15.0 statistics software (Chicago, IL, USA). A value of $\mathrm{p}<0.05$ was considered statistically significant.

\section{RESULTS}

The study included 139 consecutive adult patients with TAK, who were predominantly 
female (81.8\%) and Caucasian (83.5\%). When vessel imaging was performed, the median age and disease duration were 38.0 (27.3-47.0) years and 5.0 (1.0-12.0) years, respectively.

Table I - Demographic data, signs, symptoms and laboratory parameters when vessel imaging was requested in the 139 patients with Takayasu arteritis.

\begin{tabular}{|l|c|}
\hline Age (years) & $38.0(27.3-47.0)$ \\
Disease duration (years) & $5.0(1.0-12.0)$ \\
Female gender & $122(81.8)$ \\
Caucasian ethnicity & $116(83.5)$ \\
\hline Asymptomatic & $51(36.7)$ \\
Fatigue & $19(13.7)$ \\
Dyspnea & $15(10.8)$ \\
Weight loss & $13(9.4)$ \\
Fever & $14(10.1)$ \\
Bruit over subclavian or aorta & $15(10.8)$ \\
Carotidynia & $12(8.6)$ \\
Headache & $11(7.9)$ \\
Presyncope & $9(6.5)$ \\
Abdominal pain & $9(6.5)$ \\
Visual abnormalities & $7(5.0)$ \\
Chest pain & $5(3.6)$ \\
\hline Vessel claudication & $42(30.2)$ \\
Both upper limb & $30(21.6)$ \\
Upper left limb & $24(17.3)$ \\
Upper right limb & $17(12.2)$ \\
Both lower limb & $20(14.4)$ \\
Lower left limb & $18(12.9)$ \\
Lower right limb & $17(12.2)$ \\
\hline Increased CRP / ESR & $31(22.3)$ \\
\hline Stenosis/occlusion & \\
Right vertebral artery & $9(6.5)$ \\
Left vertebral artery & $9(6.5)$ \\
Right carotid artery & $45(32.4)$ \\
Left carotid artery & $59(42.4)$ \\
Right subclavian artery & $40(28.8)$ \\
Left subclavian artery & $72(1.8)$ \\
Ascending aorta & 0 \\
Aortic arch & $4(2.9)$ \\
Thoracic aorta & $8(5.8)$ \\
Abdominal aorta & $15(10.8)$ \\
Infrarenal aorta & $30(21.6)$ \\
Right renal artery & $30(21.6)$ \\
Left renal artery & $32(23.0)$ \\
Upper mesenteric artery & $32(23.0)$ \\
Lower mesenteric artery & $15(10.8)$ \\
Celiac trunk & $22(15.8)$ \\
Right iliac artery & $24(17.3)$ \\
Left iliac artery & $22(15.8)$ \\
\hline
\end{tabular}

Data expressed as median $\left(25^{\text {th }}-75^{\text {th }}\right.$ interquartile range) or frequency (\%). CRP: C-reactive protein; ESR: erythrocyte sedimentation rate.
Table I shows the clinical status and laboratory data, when vessel imaging was requested. One-third of the patients were asymptomatic. In this case, the imaging exams had been requested for initial diagnostic investigations (e.g., arterial pulse differences in physical examinations), and/or an increase of CRP and/or ESR. The imaging data were obtained from magnetic resonance angiography in $22(15.8 \%)$ cases, conventional angiography in $24(17.3 \%)$ cases, and computed tomography angiography in $93(66.9 \%)$ cases. The stenosis or occlusion was absent in three $(13.6 \%)$, one $(4.2 \%)$ and $10(1.1 \%)$ cases analyzed by magnetic resonance angiography, conventional angiography and computed tomography angiography, respectively.

There was no association between imaging abnormalities and demographic data (age, disease duration, gender and ethnicity), constitutional symptoms, and laboratory parameters (CRP/ESR).

Limb claudication was reported in 42 patients (30.2\%): $17.3 \%$ reported it in upper left limb (ULL), $12.2 \%$ reported it in upper right limb (URL), 12.9\% reported it in lower left limb (LLL), and $12.2 \%$ reported it in lower right limb (LRL) (Table I).

The presence or absence of limb claudication was cross-checked with vessel imaging data and the clinical status and laboratory parameters showed in Table I.

The demographic data and laboratory parameters were comparable in TAK patients with $v s$. without ULL claudication (Figure 1A). Symptoms were also similar in both groups, except for increased carotidynia $(\mathrm{p}=0.035)$, fatigue $(\mathrm{p}=0.005)$ and claudication of the URL $(\mathrm{p}<0.001)$ in TA patients with ULL claudication. Patients with ULL claudication had more left vertebral artery stenosis/occlusion $(\mathrm{p}=0.05)$, but showed no other statistically significant association, including left subclavian artery stenosis (Figure 1B).

The demographic data and laboratory parameters were also similar when comparing patients with $v s$. without URL claudication. The symptoms were also similar in both groups, except for increased claudication of the ULL $(\mathrm{p}<0.001)$, LLL $(\mathrm{p}<0.01)$ and LRL $(\mathrm{p}=0.007)$ 
Figure 1 - (A) Symptoms and laboratory parameters and (B) arterial imaging data of the Takayasu arteritis patients, according to the presence or absence of upper left limb claudication.

Legends: BO: bruit over; CRP: C-reactive protein; ESR: erythrocyte sedimentation rate; L: left; LLLC: lower left limb claudication; LRLC: lower right limb claudication; R: right; URLC: upper right limb claudication. $p$ value: $\dagger<0.05$; $\ddagger<0.001$.
Figure 2 - (A) Symptoms and laboratory parameters and $(B)$ arterial imaging data of the Takayasu arteritis patients, according to the presence or absence of upper right limb claudication.

Legends: BO: bruit over; CRP: C-reactive protein; ESR: erythrocyte sedimentation rate; L: left; LLLC: lower left limb claudication; LRLC: lower right limb claudication; R: right; ULLC: upper left limb claudication. $p$ value: $\dagger<0.05$; $\ddagger<0.001$.
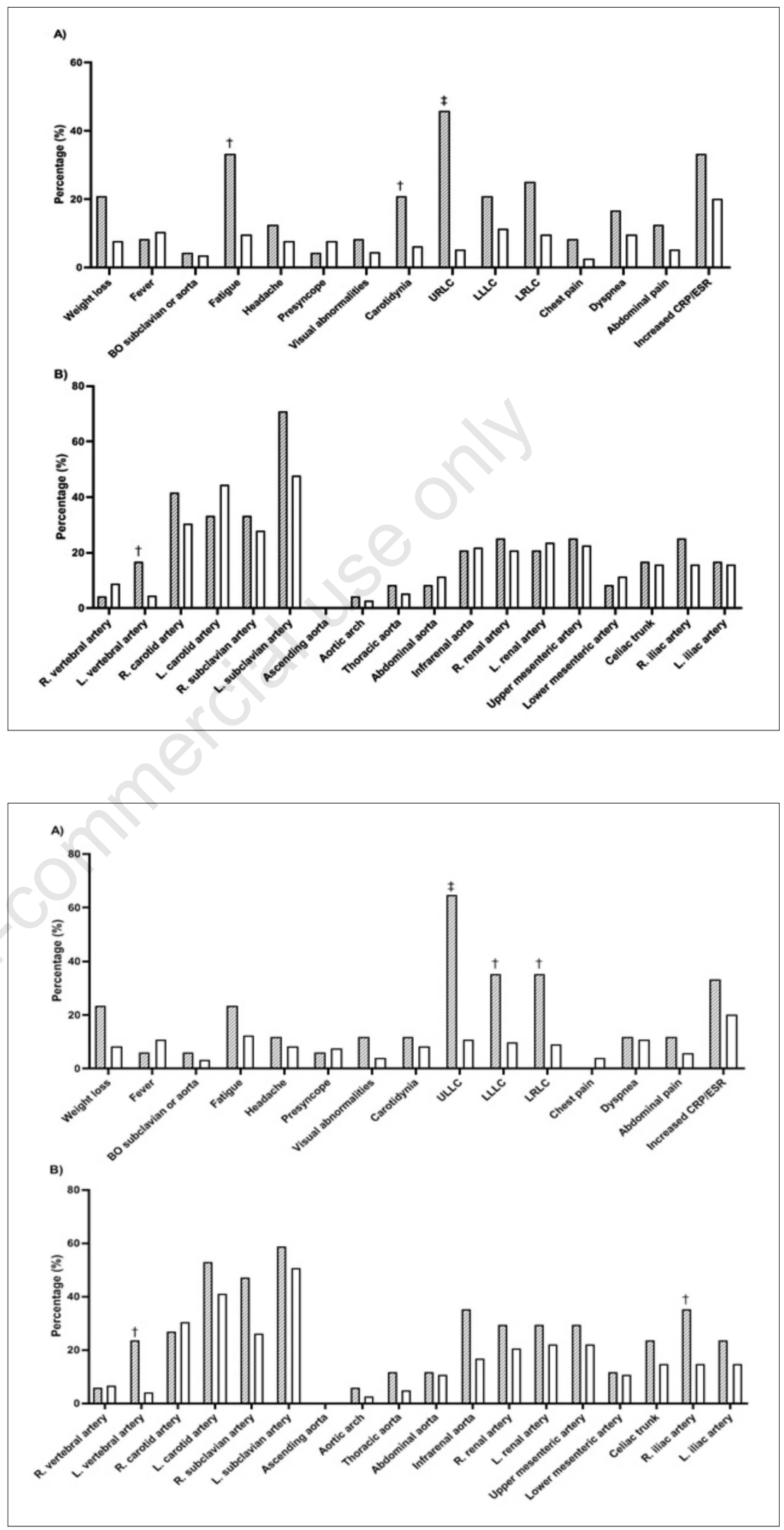

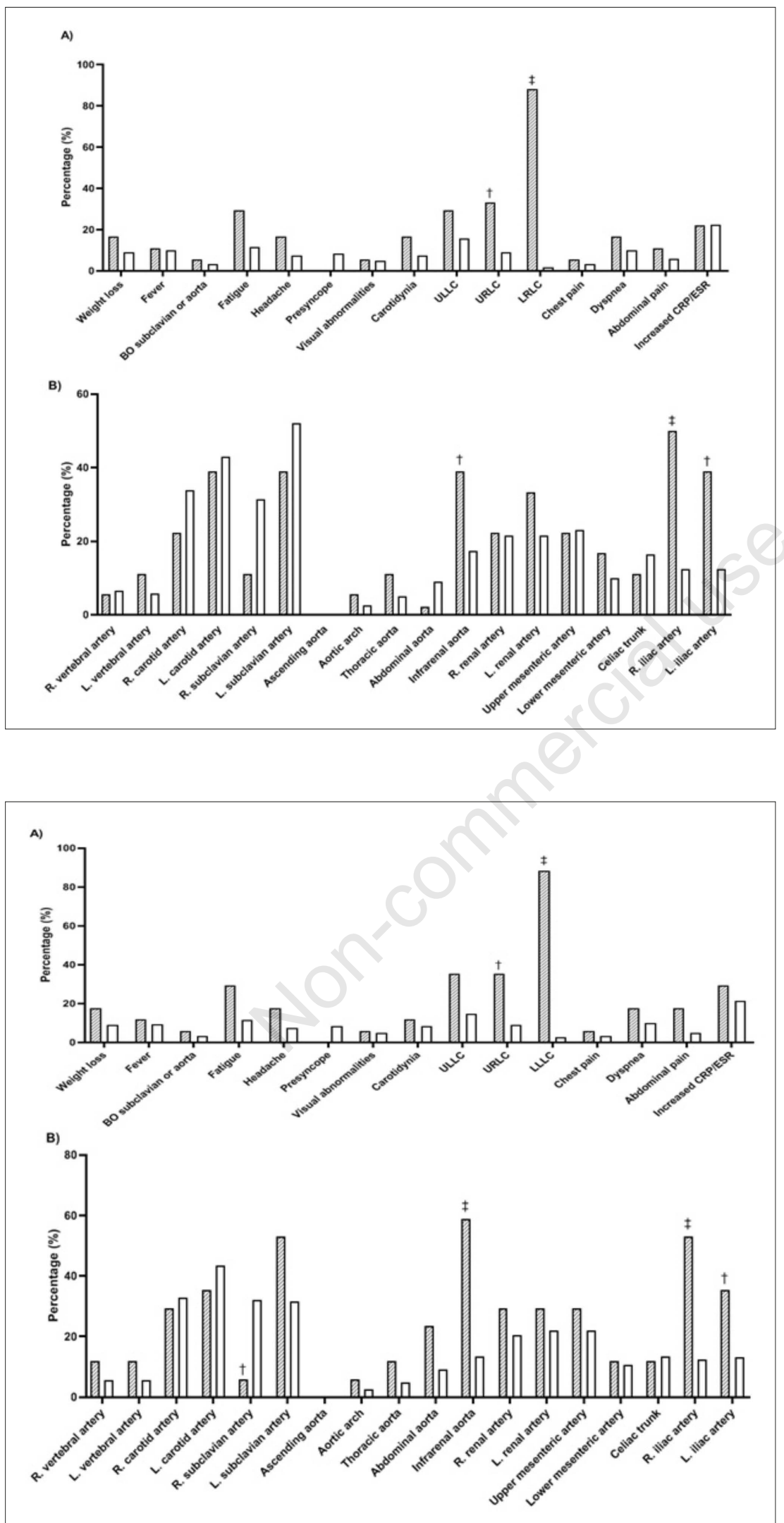

Figure 3 - (A) Symptoms and laboratory parameters and (B) arterial imaging data of the Takayasu arteritis patients, according to the presence or absence of lower left limb claudication.

Legends: BO: bruit over; CRP: C-reactive protein; ESR: erythrocyte sedimentation rate; L: left; LLLC: lower left limb claudication; R: right; ULLC: upper left limb claudication; URLC: upper right limb claudication. $p$ value: $\dagger<0.05$; $\ddagger<0.001$.

Figure 4 - (A) Symptoms and laboratory parameters and $(B)$ arterial imaging data of the Takayasu arteritis patients, according to the presence or absence of lower right limb claudication.

Legends: BO: bruit over; CRP: C-reactive protein; ESR: erythrocyte sedimentation rate; L: left; LLLC: lower left limb claudication; R: right; ULLC: upper left limb claudication; URLC: upper right limb claudication. $p$ value: $\uparrow<0.05$; $\ddagger<0.001$ 
in TA patients with URL claudication (Figure 2A). In addition, URL claudication was associated with stenosis/occlusion of the left vertebral $(\mathrm{p}=0.014)$ and right iliac $(\mathrm{p}=0.037)$ arteries, but no other associations were found, including the right subclavian artery abnormalities (Figure 2B).

Similarly, the demographic data and laboratory parameters were comparable in TAK patients with vs. without LLL claudication. Symptoms were also similar in both groups, except for increased claudication of the URL $(\mathrm{P}=0.010)$, LRL $(\mathrm{p}<0.001)$ (Figure 3A). Concerning vessel imaging, patients with LLL claudication had stenosis/occlusion of the infrarenal aorta $(\mathrm{p}=0.002)$, right iliac artery $(p<0.001)$ or left iliac artery $(p=0.007)$ (Figure $3 \mathrm{~B})$.

Finally, TAK patients with vs. without LRL claudication had similar demographic data and laboratory parameters. Symptoms were also similar in both groups, except for increased claudication in the URL ( $p$ 0.007) and LLL ( $\mathrm{p}<0.001)$ (Figure 4A). Concerning vessel imaging, patients with LRL claudication had increased right subclavian artery $(\mathrm{p}=0.024)$, infrarenal aorta $(\mathrm{p}<0.001)$, right iliac artery $(\mathrm{p}<0.001)$ or left iliac artery $(p=0.024)$ stenosis/occlusion (Figure 4B).

\section{DISCUSSION}

\section{AND CONCLUSIONS}

To the best of our knowledge, this was the first study to associate upper and lower limb claudication with imaging studies in patients with TAK. In this study the imaging findings in patients with upper limb claudication did not justify it through an ischemic mechanism; therefore they showed a clinical-radiographic dissociation regarding claudication of the upper limbs. The same was not shown for lower limbs. Moreover, the patients who had claudication of one limb were more prone to having the same symptoms in other limbs and to having more fatigue, which may suggest a central pain pathway for this symptom in patients with TAK.

Although TAK is a rare systemic vasculitis, this analysis included a sample of 139 patients, which is more than most published observational studies of TAK. In addition, in- formation on patients was based on previously parameterized data, which ensured reliable study data.

Limb claudication was present in one-third of our patients. This frequency was similar to other reports from Brazil (15), but it was inferior to the frequency reported in North America and Europe (16-18).

In this study, upper limb claudication was statistically associated only with left vertebral artery SAS, but not to the subclavian arteries, suggesting a clinical-radiographic dissociation. This phenomenon has been extensively shown in osteo-muscular disorders, such as fibromyalgia (19), lower back pain (20) and osteoarthritis (21), but it has not been yet studied in TAK. However, this clinical-radiographic dissociation does not seem to occur often in other cases of limb claudication such as peripheral arterial disease (22).

There are no studies that fully explain the pathophysiology of limb claudication in TAK. Intermittent claudication in peripheral arterial disease not caused by vasculitis is explained by differing degrees of arterial occlusion and critical ischemia of extremities (23, 24). However, this concept cannot be extrapolated to TAK, because there are some clinical and anatomical differences. For example, TAK predominantly affects upper limbs and causes proximal arterial narrowing or occlusion of the aorta and its main branches, whereas peripheral arterial disease most often affects the lower limbs and in a distal fashion $(7,22-25)$.

Vessel narrowing leading to limb ischemia did not seem to fully explain the claudication of upper limbs in patients with TAK. Moreover, we found that patients who had limb claudication in one limb were more likely to have the same symptom in another, and there was a statistical association between limb claudication and fatigue. These findings perhaps point to a relationship between limb claudication in TAK patients and central pain sensitization disorders, such as fibromyalgia (19). Alibaz-Oner et al. (19) reported a similar frequency of fibromyalgia between the general population and patients with TAK, but in the subset of active TAK patients the incidence of fibromyalgia was significantly 
higher. However, this study did not examine limb claudication data.

Conversely, we did not find an association between limb claudication and demographic data, elevated CRP/ESR or other symptoms usually related to TAK activity. This suggests that the imaging studies were performed in patients whose diseases were probably not active. This makes us question the validity of pursuing limb claudication as a marker of disease activity in TAK.

As for the limitations of our study, it was retrospective and performed in a single center. In addition, follow-up imaging was not performed to check whether arterial narrowing eventually ensued in the claudicating limb. The vessel imaging methods were not standardized (different methods were applied) and usually only evaluated the aorta and its main branches, so no evaluation of distal arterial narrowing was performed. Finally, we did not analyze the possible effect of treatment on patients' symptoms.

The present study shows a need to reassess the concept of upper limb claudication as a clinical sign of arterial narrowing, and perhaps rethink the use of imaging methods in TAK patients with limb claudication. More data is necessary to understand the pathophysiology of TAK symptoms and its relation to other central sensitization syndromes.

\section{Funding}

This work was funded by Fundação de Amparo à Pesquisa do Estado de São Paulo (FAPESP): \#2018/08735-3 to AMS; Conselho Nacional de Desenvolvimento Científico e Tecnológico (CNPq) 303379/2018-9, and Faculdade de Medicina da USP to SKS.

\section{Conflicts of interest}

The authors declare that they have no conflicts of interest.

\section{REFERENCES}

1. Kerr GS, Hallahan CW, Giordano J, et al. Takayasu arteritis. Ann Intern Med. 1994; 120: 919-9.

2. Kerr GS. Takayasu's arteritis. Rheum Dis Clin North Am. 1995; 21: 1014-58.

3. Numano F, Kobayashi Y. Takayasu arteritis - beyond pulselessness. Int Med. 1999; 3: 226-32.
4. Hall S, Barr W, Lie JT, et al. Takayasu arteritis. A study of 32 North American patients. Medicine. 1985; 64: 89-9.

5. Jennette JC, Falk RJ, Bacon PA, et al. 2012 revised International Chapel Hill Consensus Conference Nomenclature of vasculitides. Arthritis Rheum. 2013; 65: 1-11.

6. Hunder GG, Arend WP, Bloch DA, et al. The American College of Rheumatology 1990 criteria for the classification of vasculitis: Introduction. Arthritis Rheum. 1990; 33: 1065-7.

7. Hata A, Noda M, Moriwaki R, Numano F. Angiographic findings of Takayasu arteritis: New classification. Int J Cardiol. 1996; 54: 155-63.

8. Yamato M, Lecky JW, Hiramatsu K, Kohda E. Takayasu arteritis: radiographic and angiographic findings in 59 patients. Radiology. 1986; 161: 329-34.

9. Jennette JC, Falk RJ, Andrassy K, et al. Nomenclature of systemic vasculitides. Arthritis Rheum. 1994; 37: 187-92.

10. Dormandy J, Heeck L, Vig S. The natural history of claudication: risk to life and limb. Semin Vasc Surg. 1999; 12: 123-37.

11. Cockett FB, Maurice BA. The ischaemic leg. Br Med J. 1965; 1: 1137-8.

12. Mason JC. Takayasu arteritis-advances in diagnosis and management. Nat Rev Rheumatol. 2010; 6: 406-15.

13. Rizzi R, Bruno S, Stellacci C, Dammacco R. Takayasu's arteritis: a cell-mediated largevessel vasculitis. Int J Clin Lab Res. 1999; 29: 8-13.

14. Nemcek AA, Bhave A. Diagnostic evaluation of aortoiliac occlusive disease: when is a lesion significant? Techn Vasc Interv Radiol. 2000; 3: 180-5.

15. Sato EI, Lima DNS, Santo BE, Hata F. Takayasu arteritis: Treament and prognosis in a University Center in Brazil. Int J Cardiol. 2000; 75: S163-6.

16. Hall S, Barr W, Lie JT, et al. Takayasu's arteritis - A study of 32 North American patients. Medicine (Baltimore). 1985; 64: 89-9.

17. Vanoli, M, Daina E, Salvarani C, et al. Takayasu's arteritis: A study of 104 Italian patients. Arthritis Rheum. 2005; 53: 100-7.

18. Bilge NSY, Kaşifoğlu T, Cansu DU, Korkmaz C. Retrospective evaluation of 22 patients with Takayasu's arteritis. Rheumatol Int. 2012; 32: 1155-9.

19. Alibaz-Oner F, Can M, Birkan I, et al. Presence of fibromyalgia in patients with Takayasu's arteritis. Intern Med. 2013; 52: 273942.

20. HumphreYS SC, Eck JC, Hodges SD. Neuroimaging in low back pain. Am Fam Physician. 2002; 65: 2299-306.

21. Link TM, Steinbach LS, Ghosh S, et al. Osteoarthritis: MR imaging findings in different 
stages of disease and correlation with clinical findings. Radiology. 2003; 226: 373-81.

22. Hamburg NM, Creager MA. Pathophysiology of intermittent claudication in peripheral artery disease. Circ J. 2017; 81: 281-9.

23. Uccioli L, Meloni M, Izzo V, et al. Critical limb ischemia: current challenges and future pros- pects. Vasc Health Risk Manag. 2018; 14: 63-74. 24. Seyahi E. Takayasu arteritis: an update. Curr Opin Rheumatol. 2017; 29: 51-56.

25. Mavor GE. The pattern of occlusion in atheroma of the lower limb arteries the correlation of clinical and arteriographic findings. Br J Surgery. 1956 ; 43: 352-64. 